\title{
Effect of annealing atmosphere on the electrophysical properties of $\mathrm{Sr}_{2} \mathrm{CuO}_{3}$
}

\author{
I. B. Bobylev, S. V. Naumov ${ }^{\dagger}$, N. A. Zyuzeva \\ †naumov@imp.uran.ru
}

\author{
M. N. Miheev Institute of Metal Physics UB RAS, Ekaterinburg, 620108, Russia
}

\begin{abstract}
The effect of the annealing atmosphere on the electrophysical properties (electrical conductivity and thermo-EMF) of strontium orthocuprate $\left(\mathrm{Sr}_{2} \mathrm{CuO}_{3}\right)$ at temperatures up to $150^{\circ} \mathrm{C}$ was studied. At temperatures of $100-150^{\circ} \mathrm{C}$, depending on the annealing atmosphere, strontium orthocuprate is capable of absorbing hydrogen and water. Intercalation of both hydrogen and water leads to the appearance of ionic conductivity in $\mathrm{Sr}_{2} \mathrm{CuO}_{3}$. In the case of sample processing in a hydrogen atmosphere, new positive current carriers $\left(\mathrm{H}^{+}\right)$appear, while during processing in a humid atmosphere, the current carriers are the products of water dissociation - both positive $\left(\mathrm{H}^{+}\right)$and negative $\left(\mathrm{OH}^{-}\right)$ions. The conductivity of strontium orthocuprate decreases as hydrogen is absorbed. The reason is a decrease in the mobility of the main current carriers (holes) due to an increase of the lattice defect due to the introduction of hydrogen ions into it. When hydrogen or a humid atmosphere replaces the oxygen atmosphere of annealing, a sharp change in the electrophysical properties is observed. Intercalation of hydrogen or water dissociation products into the $\mathrm{Sr}_{2} \mathrm{CuO}_{3}$ structure indicates a high mobility of these ions at temperatures above $70^{\circ} \mathrm{C}$. The appearance of additional ions $\left(\mathrm{H}^{+}\right.$and $\left.\mathrm{OH}^{-}\right)$in the structure of $\mathrm{Sr}_{2} \mathrm{CuO}_{3}$, which are structural defects, leads to a decrease in the mobility of the main of current carriers. Thus, it was shown that strontium orthocuprate is a mixed-type semiconductor. At temperatures below $70^{\circ} \mathrm{C}, \mathrm{Sr}_{2} \mathrm{CuO}_{3}$ is an n-type semiconductor, and at higher temperatures $\left(100-150^{\circ} \mathrm{C}\right)$ it becomes a p-type semiconductor.
\end{abstract}

Keywords: strontium orthocuprate, electrical conductivity, thermo-EMF.

УДК: 541.67

\section{Влияние атмосферы отжига на электрофизические свойства $\mathrm{Sr}_{2} \mathrm{CuO}_{3}$}

\author{
Бобылев И.Б., Наумов С. В. ${ }^{\dagger}$, Зюзева Н. А. \\ Институт физики металлов имени М.Н. Михеева УрО РАН, Екатеринбург, 620108, Россия
}

Исследовано влияние атмосферы отжига на электрофизические свойства (электропроводность и термо-ЭДС) ортокупрата стронция $\mathrm{Sr}_{2} \mathrm{CuO}_{3}$ при температурах до $150^{\circ} \mathrm{C}$. При температурах $100-150^{\circ} \mathrm{C}$ в зависимости от атмосферы отжига ортокупрат стронция способен поглощать водород и воду. Интеркаляция как водорода, так и воды приводит к появлению в $\mathrm{Sr}_{2} \mathrm{CuO}_{3}$ ионной проводимости. В случае обработки образцов в атмосфере водорода появляются новые положительные носители тока $\left(\mathrm{H}^{+}\right)$, в то время как при обработке во влажной атмосфере носителями тока являются продукты диссоциации воды - как положительные $\left(\mathrm{H}^{+}\right)$, так и отрицательные $\left(\mathrm{OH}^{-}\right)$ионы. По мере поглощения водорода электропроводность ортокупрата стронция снижается. Причиной тому является уменьшение подвижности основных носителей тока (дырок) вследствие увеличения дефектности решетки в связи с внедрением в нее ионов водорода. При смене окислительной атмосферы отжига на водород или влажную атмосферу наблюдается резкое изменение электрофизических свойств, которое связано с проникновением водорода или продуктов диссоциации воды в структуру $\mathrm{Sr}_{2} \mathrm{CuO}_{3}$, что свидетельствует о высокой подвижности этих ионов при температурах выше $70^{\circ} \mathrm{C}$. Появление в структуре $\mathrm{Sr}_{2} \mathrm{CuO}_{3}$ дополнительных ионов $\left(\mathrm{H}^{+}\right.$и $\left.\mathrm{OH}^{-}\right)$, являющихся структурными дефектами, приводит к снижению подвижности основных носителей тока. Таким образом, показано, что ортокупрат стронция является полупроводником смешанного типа. При температурах, не превышающих $70^{\circ} \mathrm{C}, \mathrm{Sr}_{2} \mathrm{CuO}_{3}$ является полупроводником n-типа, а при более высоких температурах $\left(100-150^{\circ} \mathrm{C}\right)$ он становится полупроводником р-типа.

Ключевые слова: ортокупрат стронция, электропроводность, термо-ЭДС. 


\section{1. Введение}

Интерес исследователей к перовскитоподобному ортокупрату стронция $\left(\mathrm{Sr}_{2} \mathrm{CuO}_{3}\right)$ вызывает тот факт, что при переходе в определенных условиях в тетрагональную фазу он становится сверхпроводником с критической температурой, достигающей 100 K $[1,2]$. Также известно, что в ряде оксидов, в том числе в перовскитоподобных, при внедрении в их структуру водорода возникает протонная проводимость $[3,4]$. Это дает основание предполагать, что ионной проводимостью могут обладать и купраты со структурой перовскита, в которых обнаружены признаки протонной проводимости при относительно низких температурах [5-7]. Водород действует как донор и может существенно повлиять на электрические свойства оксидов [8]. В работе [9] показано, что введенный водород не только компенсирует недостаток кислорода, но и создает дефекты в оксидных соединениях. В [10] было показано, что $\mathrm{Sr}_{2} \mathrm{CuO}_{3}$ при температуре $150^{\circ} \mathrm{C}$ поглощает $0.12 \%$ водорода, что соответствует формуле $\mathrm{H}_{0.35} \mathrm{Sr}_{2} \mathrm{CuO}_{3}$.

Ранее [11] нами было исследовано влияние паров воды на электропроводность ортокупрата стронция. Было установлено, что взаимодействие $\mathrm{Sr}_{2} \mathrm{CuO}_{3}$ с влажной атмосферой при $T=150^{\circ} \mathrm{C}$ приводит к интеркаляции в его структуру ионов водорода и гидроксила. Внедрение этих ионов может являться причиной появления ионной проводимости. Ортокупрат стронция после синтеза при атмосферном давлении находится в орторомбической фазе, но в отличие от $\mathrm{YBa}_{2} \mathrm{Cu}_{3} \mathrm{O}_{y}$ не обладает сверхпроводимостью. Отжиг $\mathrm{YBa}_{2} \mathrm{Cu}_{3} \mathrm{O}_{y}$ во влажной атмосфере значительно ускоряет процесс диффузии кислорода в образец [12]. Взаимодействие влажного воздуха с перовскитоподобными соединениями может проходить по разным сценариям: как формирование дефектной структуры по всему объему вещества [12], так и по границам зерен [13]. В более сложных перовскитоподобных оксидных соединениях, в частности, в легированном стронцием и магнием галлате лантана, который представляет собой твердый кислород-проводящий электролит, в поверхностных слоях образцов обнаружены $\mathrm{SrO}$ и гидрооксид лантана, но это происходит в процессе высокотемпературной обработки в атмосфере $\mathrm{CO}_{2}[14]$.

Целью настоящей работы является исследование электропроводности и термо-ЭДС ортокупрата стронция в зависимости от температуры и характера атмосферы отжига. Термоэлектрические измерения позволяют определять тип носителей тока, а также их концентрацию и подвижность [15]. Применение этого метода может позволить установить механизм влияния водорода и паров воды на электропроводность $\mathrm{Sr}_{2} \mathrm{CuO}_{3}$ и показать, что внедрение в его структуру ионов водорода или гидроксила является причиной возникновения в нем ионной проводимости.

\section{2. Экспериментальная часть}

В качестве материала для исследования использовали $\mathrm{Sr}_{2} \mathrm{CuO}_{3}$, полученный по стандартной керамической технологии [10]. В качестве исходных материалов для синтеза $\mathrm{Sr}_{2} \mathrm{CuO}_{3}$ использовали $\mathrm{SrCO}_{3}$ и $\mathrm{CuO}$ марки ос. ч. Образцы для измерения электропроводности и термо-ЭДС были спрессованы при давлении 10 МПа в виде брусков с размерами $\sim 18 \times 3 \times 2$ мм. Спекание их проводили при температуре $950^{\circ} \mathrm{C}$. Аттестацию образцов на плотность и пористость провели в соответствии с ГОСТ 2409-95 методом гидростатического взвешивания. Плотность образцов составила $3.77 \mathrm{r} / \mathrm{cm}^{3}$ или $69 \%$ от рентгеновской плотности, открытая пористость 28\%. Рентгенофазовый анализ образцов выполнен на дифрактометре «Empyrean» фирмы PANalytical в медном излучении $\left(\mathrm{Cu}_{\mathrm{Ka}}\right)$. Анализ показал, что все образцы являются однофазными. Для измерения электропроводности использовали четырехконтактный метод. Измерения проводили при температурах $40-150^{\circ} \mathrm{C}$ в атмосфере сухого воздуха, водорода или во влажной атмосфере. Выбор температурного диапазона основан на наших предварительных исследованиях и обусловлен тем, что при более высоких температурах именно в атмосферах, содержащих влагу, $\mathrm{Sr}_{2} \mathrm{CuO}_{3}$ деградирует. Подобные результаты, указывающие на разложение $\mathrm{Sr}_{2} \mathrm{CuO}_{3}$ в парах воды при температурах выше $150^{\circ} \mathrm{C}$ с образованием соединения $\mathrm{Sr}_{2} \mathrm{Cu}(\mathrm{OH})_{6}$, получены также другими авторами [16]. Водород получали с помощью генератора водорода «Спектр-6», снабженного трехступенчатой системой очистки. Содержание воды в нем не превышало $0.001 \%$. Измерение коэффициента термо-ЭДС образцов проводили на естественном температурном градиенте печи, величину которого определяли прижатыми к образцу двумя термопарами ТПП10. Возникающую при этом разность потенциалов (термо-ЭДС) с платиновых контактов термопар измеряли при помощи вольтметра, в качестве которого использовали специализированную плату расширения для регулятора Zirconia M. Полученное значение термоЭДС корректировали на термо-ЭДС платины [17].

\section{3. Результаты и обсуждение}

Результаты измерений показали, что в атмосфере сухого воздуха электропроводность $\mathrm{Sr}_{2} \mathrm{CuO}_{3}$ экспоненциально растет с повышением температуры (Рис. 1, кривая 1). Значения термо-ЭДС ниже $\sim 70^{\circ} \mathrm{C}$ имеют отрицательный знак, что совпадает с [1]. При более высокой температуре знак меняется на положительный и с ростом температуры термо-ЭДС продолжает увеличиваться (Рис. 2, кривая 1). Таким образом, $\mathrm{Sr}_{2} \mathrm{CuO}_{3}$ является полупроводником смешанного типа. При температурах, близких к комнатной, основными носителями тока являются электроны, но при повышении температуры число дырок увеличивается, и они становятся основными носителями, что характерно для полупроводников [18].

Отжиг при температуре $150^{\circ} \mathrm{C}$ в сухом воздухе показал, что значения электропроводности и термоЭДС не изменялись. При введении водорода в атмосферу отжига электропроводность $\mathrm{Sr}_{2} \mathrm{CuO}_{3}$ стала уменьшаться (Рис. 3, кривая 1). Через непродолжительное время (0.5 час.) электропроводность несколько увеличилась, на временной зависимости появился максимум, после 
чего электропроводность продолжила монотонно снижаться. Падение электропроводности можно связать с уменьшением подвижности дырок вследствие появления структурных дефектов, которыми в данном случае являются ионы водорода.

При замене воздушной атмосферы на водород величина термо-ЭДС в течение получаса оставалась постоянной. Затем термо-ЭДС резко выросла и продолжала слабо увеличиваться при продолжении отжига (Рис. 4, кривая 1). Резкий рост термо-ЭДС за короткий промежуток времени указывает на то, что в ходе замены атмосферы в $\mathrm{Sr}_{2} \mathrm{CuO}_{3}$ появляются новые положительные носители, которыми могут являться только ионы водорода. Обращает на себя внимание

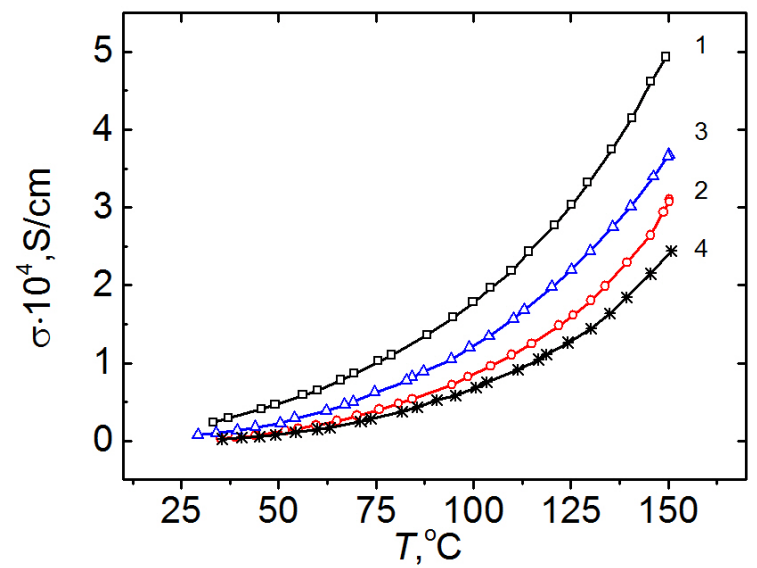

Pис. 1. (Color online) Температурные зависимости электропроводности $\mathrm{Sr}_{2} \mathrm{CuO}_{3}$ в различных атмосферах: 1-в сухом воздухе, 2 - в атмосфере водорода; 3 - в насыщенном парами воды воздухе; 4-в насыщенном парами воды водороде.

Fig. 1. (Color online) Temperature dependences of the electrical conductivity of $\mathrm{Sr}_{2} \mathrm{CuO}_{3}$ in different atmospheres: 1 - in dry air, 2 - in a hydrogen atmosphere; 3 - in water vapor-saturated air; 4 - in water vapor-saturated hydrogen.

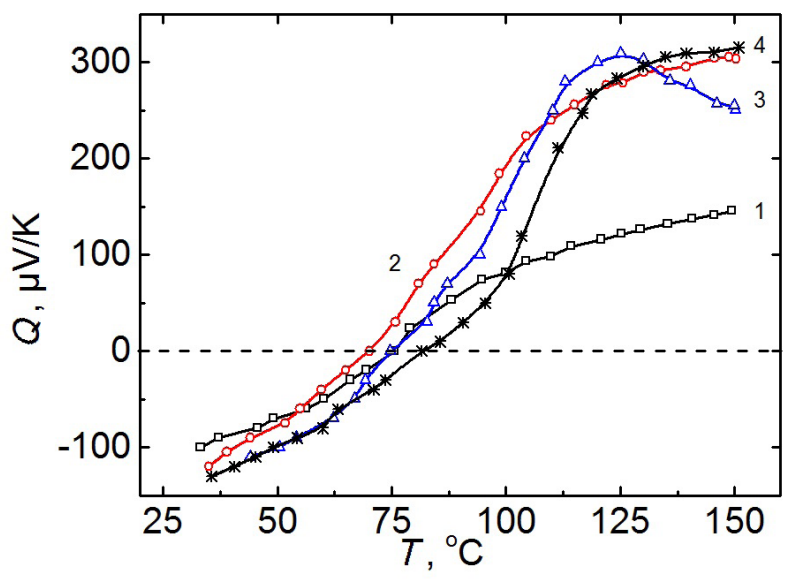

Pис. 2. (Color online) Температурные зависимости термо-ЭДС $\mathrm{Sr}_{2} \mathrm{CuO}_{3}$ в различных атмосферах: 1 - в сухом воздухе, 2 - в атмосфере водорода; 3 - в насыщенном парами воды воздухе; 4 - в насыщенном парами воды водороде.

Fig. 2. (Color online) Temperature dependences of thermo-EMF of $\mathrm{Sr} 2 \mathrm{CuO} 3$ in different atmospheres: 1 - in dry air, 2-in hydrogen atmosphere; 3 - in water vapor-saturated air; $4-$ in water vaporsaturated hydrogen. тот факт, что максимум на временной зависимости электропроводности совпадает по времени с резким ростом термо-ЭДС (Рис. 3,4, кривые 1). По-видимому, при смене атмосферы воздуха на водород в результате роста концентрации ионов водорода в течение получаса достигается порог протонной проводимости, что и является причиной появления максимума на временной зависимости электропроводности. Однако при дальнейшем отжиге электропроводность продолжала падать, т.к. характер ее временной зависимости, вероятно, определяется снижением подвижности основных дырочных носителей. Наличие максимумов на кривых 1 (Рис. 3,4) свидетельствуют о том, что увеличение числа положительных носителей на опре-

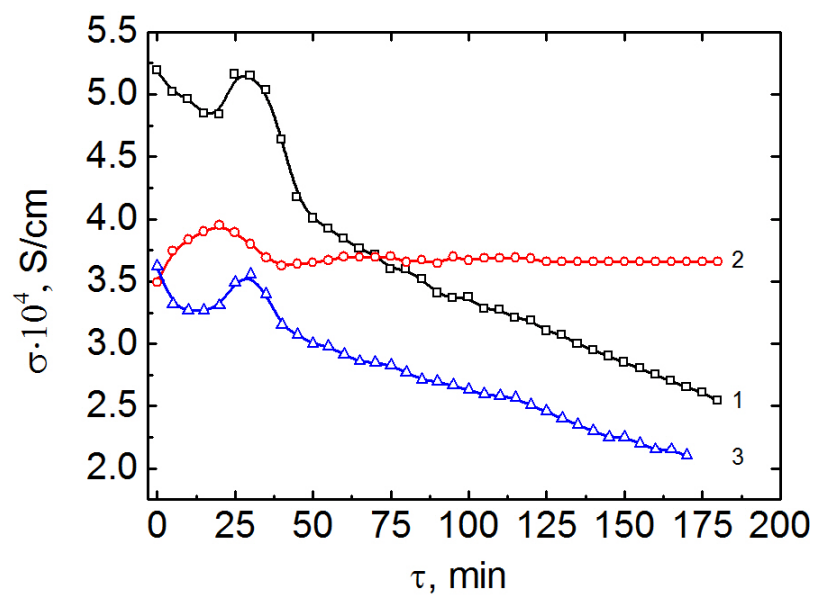

Pис. 3. (Color online) Зависимости электропроводности $\mathrm{Sr}_{2} \mathrm{CuO}_{3}$ от времени отжига при $T=150^{\circ} \mathrm{C}: 1-$ в атмосфере водорода; 2 - в насыщенном парами воды воздухе; 3 - в насыщенном парами воды водороде.

Fig. 3. (Color online) Dependence of the electrical conductivity of $\mathrm{Sr}_{2} \mathrm{CuO}_{3}$ at $T=150^{\circ} \mathrm{C}$ on the annealing time: $1-$ in a hydrogen atmosphere; 2 - in water vapor-saturated air; 3 - in water vaporsaturated hydrogen.

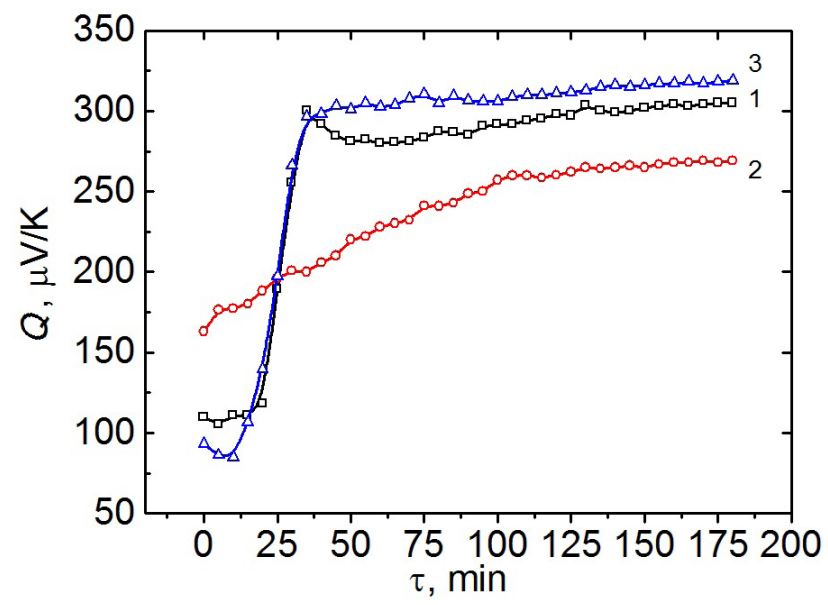

Puc. 4. (Color online) Зависимости термо-ЭДС $\mathrm{Sr}_{2} \mathrm{CuO}_{3}$ от времени отжига при $T=150^{\circ} \mathrm{C}: 1-$ в атмосфере водорода; 2 - в насыщенном парами воды воздухе; 3 - в насыщенном парами воды водороде.

Fig. 4. (Color online) Dependences of thermo-EMF of $\mathrm{Sr}_{2} \mathrm{CuO}_{3}$ at $T=150^{\circ} \mathrm{C}$ on the annealing time: $1-$ in a hydrogen atmosphere; $2-$ in water vapor-saturated air; $3-$ in water vapor-saturated hydrogen. 
деленном временном промежутке вносит больший вклад в электропроводность, чем падение подвижности дырок.

Согласно предыдущей нашей работе [11], ЭДС электрохимической ячейки с использованием $\mathrm{Sr}_{2} \mathrm{CuO}_{3}$, также возникает в течение того же промежутка времени при замене воздушной атмосферы на водород. Совпадение максимума по времени на зависимости электропроводности с резким ростом термо-ЭДС и появлением электрохимической ЭДС свидетельствует о том, что причиной всех этих эффектов является возникновение протонной проводимости. Резкое изменение свойств $\mathrm{Sr}_{2} \mathrm{CuO}_{3}$ говорит о высокой подвижности ионов водорода при $T=150^{\circ} \mathrm{C}$.

Известно [15], что наличие дефектов структуры, которыми являются атомы, отличающиеся по размеру от атомов основной фазы, приводит к уменьшению подвижности дырок. Поэтому наиболее вероятной причиной снижения электропроводности с увеличением времени отжига в атмосфере водорода является внедрение протона, который является таким структурным дефектом. Очевидно, что с увеличением времени термообработки число ионов водорода растет. Тем не менее, вклад снижения подвижности дырок в проводимость превалирует над вкладом роста числа ионов водорода по причине значительно меньшей подвижности протона по сравнению с подвижностью дырок.

Из температурной зависимости электропроводности в атмосфере водорода видно, что проводимость гидрированного $\mathrm{Sr}_{2} \mathrm{CuO}_{3}$ растет по мере повышения температуры (Рис. 1, кривая 2). Эта зависимость подобна зависимости, полученной в атмосфере сухого воздуха. Подобное наблюдается и для температурных зависимостей термо-ЭДС, полученных в атмосфере водорода и сухого воздуха (Рис. 2, кривые 1,2). При $T<70^{\circ} \mathrm{C}$ в гидрированном $\mathrm{Sr}_{2} \mathrm{CuO}_{3}$ основными носителями остаются электроны. С ростом температуры возрастает число дырок, а также число и подвижность ионов водорода. При этом, несмотря на большее значение термо-ЭДС, электропроводность образца в атмосфере водорода при $T=150^{\circ} \mathrm{C}$ заметно меньше, чем в атмосфере сухого воздуха. Это может быть также следствием снижения подвижности дырок из-за присутствия в структуре $\mathrm{Sr}_{2} \mathrm{CuO}_{3}$ ионов водорода.

После обратной замены атмосферы водорода на сухой воздух был проведен отжиг образца при $T=150^{\circ} \mathrm{C}$. Значение проводимости оставалось постоянным, но оно было существенно ниже, чем для исходного образца и близко к значению, характерному для отжига в атмосфере водорода. При этом величина термо-ЭДС в 1.5 раза превышала значение, полученное при отжиге в сухом воздухе. Это свидетельствует о неполной обратимости свойств ортокупрата стронция при изменении характера атмосферы. Возможно, необратимость связана с тем, что после гидрирования в $\mathrm{Sr}_{2} \mathrm{CuO}_{3}$ сохраняются структурные дефекты, связанные с неполным удалением водорода при температуре $150^{\circ} \mathrm{C}$. Кроме того, в $[10,19]$ было показано, что гидрирование более сложных купратов, таких как $\mathrm{YBa}_{2} \mathrm{Cu}_{3} \mathrm{O}$, приводит к снижению температуры поглощения кислорода с 350 до $\sim 150^{\circ} \mathrm{C}$, что также может быть причиной необратимости электрофизических свойств при изменении характера атмосферы.

При замене сухой воздушной атмосферы на влажную на временной зависимости проводимости $\mathrm{Sr}_{2} \mathrm{CuO}_{3}$ в течение получаса также появился максимум (Рис. 3, кривая 2). При этом термо-ЭДС существенно выросла (Рис. 4, кривая 2), что указывает на появление новых носителей тока. Ранее $[11,20]$ было установлено, что при термообработке при $150^{\circ} \mathrm{C}$ во влажной атмосфере $\mathrm{Sr}_{2} \mathrm{CuO}_{3}$ поглощает воду, которая, диссоциируя на поверхности кристаллита, приводит к появлению в его структуре ионов водорода и гидроксила [21]. Их число со временем отжига возрастает, однако, проводимость после максимума в ходе отжига при $T=150^{\circ} \mathrm{C}$ оставалась практически постоянной. Это может быть связано с одновременным протеканием двух процессов, вносящих противоположные вклады в электропроводность, так как наряду с ростом числа ионов водорода и гидроксила снижается подвижность дырок.

Температурная зависимость электропроводности $\mathrm{Sr}_{2} \mathrm{CuO}_{3}$ в атмосфере воздуха, насыщенного парами воды, подобна соответствующей зависимости, полученной в атмосфере водорода (Рис. 1, кривая 3). Однако в отличие от обработки в сухом воздухе или в водороде, термо-ЭДС с ростом температуры выше $120^{\circ} \mathrm{C}$ слабо уменьшалась (Рис. 2, кривая 3). По-видимому, этот результат является следствием того, что при поглощении воды в $\mathrm{Sr}_{2} \mathrm{CuO}_{3}$ внедряются как положительные, так и отрицательные носители тока, которые вносят разный вклад в термо-ЭДС.

Замена при $T=150^{\circ} \mathrm{C}$ влажного воздуха на влажный водород в течение получаса привела к заметному падению электропроводности (Рис. 3, кривая 3). А далее, так же, как это происходило при замене сухого воздуха на водород, на временной зависимости электропроводности появился максимум. При этом величина термо-ЭДС резко увеличилась (Рис. 4, кривая 3). Это еще раз свидетельствует о том, что с появлением в атмосфере отжига водорода число положительных носителей в ортокупрате стронция существенно возрастает. Как и в случае замены сухого воздуха на водород, увеличение числа положительных носителей тока при смене влажного воздуха на влажный водород сопровождается снижением электропроводности вследствие появления структурных дефектов (ионов водорода).

Зависимость электропроводности $\mathrm{Sr}_{2} \mathrm{CuO}_{3}$ от температуры во влажном водороде подобна аналогичной зависимости в сухом водороде (Рис. 1, кривая 4). Термо-ЭДС также возрастает в связи с ростом числа положительных носителей тока (Рис. 2, кривая 4), но при $T<70^{\circ} \mathrm{C} \quad \mathrm{Sr}_{2} \mathrm{CuO}_{3}$ по-прежнему остается полупроводником n-типа. Это говорит о том, что при температурах до $70^{\circ} \mathrm{C}$ подвижность ионов водорода и гидроксила очень мала, и основными носителями являются электроны. Интеркаляция в $\mathrm{Sr}_{2} \mathrm{CuO}_{3}$ как водорода, так и воды приводит к уменьшению электропроводности, причем водород влияет сильнее, чем вода. Однако значения термо-ЭДС при $T>100^{\circ} \mathrm{C}$ зна- 
чительно выше по сравнению со значениями, полученными в атмосфере сухого воздуха, что может быть связано с присутствием нескольких типов носителей тока.

Величина электропроводности определяется изменением подвижности основных носителей тока. Однако на временных зависимостях электропроводности в атмосфере водорода или во влажной атмосфере наблюдаются максимумы (Рис. 3). При интеркалировании в $\mathrm{Sr}_{2} \mathrm{CuO}_{3}$ воды или водорода возникает сложная структура, в которой при $t>70^{\circ} \mathrm{C}$ основными носителями являются дырки, к которым в процессе гидрирования добавляются ионы водорода, а при гидратировании - водорода и гидроксила.

В обоих случаях протон может присоединиться к кислороду с образованием иона гидроксила, либо разместиться в междоузлиях. В первом случае в системе возникают дополнительные отрицательные носители тока $\left(\mathrm{OH}^{-}\right)$, а во втором, кроме ионов гидроксила - новые положительные носители $\left(\mathrm{H}^{+}\right)$. В [22] предположили, что протон может размещаться в междоузлиях. Имеются доказательства того, что он входит в структуру купратов без образования гидроксильных групп [5]. Однако вхождение водорода в структуру $\mathrm{Sr}_{2} \mathrm{CuO}_{3}$ не приводит к появлению в образцах элементарной меди, в то время как $\mathrm{CuO}$ при $T \sim 110^{\circ} \mathrm{C}$ полностью восстанавливается водородом [23].

Если поглощение воды приводит к росту параметров решетки $\mathrm{Sr}_{2} \mathrm{CuO}_{3}$ [20], то после поглощения водорода параметры решетки снижались [10]. Различия между гидратированием и гидрированием могут свидетельствовать о том, что в случае воды образуются ионы водорода и гидроксила, а при поглощении водорода - только ионы водорода. Последние размещаются в междоузельных позициях, а ионы гидроксила могут размещаться в имеющихся в структуре $\mathrm{Sr}_{2} \mathrm{CuO}_{3}$ кислородных вакансиях. В обоих случаях ромбическое искажение решетки слабо меняется и $\mathrm{Sr}_{2} \mathrm{CuO}_{3}$ не переходит в тетрагональную фазу $[10,20]$.

\section{4. Заключение}

Ортокупрат стронция является полупроводником смешанного типа. При $T<70^{\circ} \mathrm{C}$ он является полупроводником n-типа, а при более высоких температурах он становится полупроводником р-типа.

При температурах $100-150^{\circ} \mathrm{C}$ в зависимости от атмосферы отжига $\mathrm{Sr}_{2} \mathrm{CuO}_{3}$ способен поглощать водород и воду. Интеркаляция как водорода, так и воды приводит к появлению в нем ионной проводимости. В случае обработки в атмосфере водорода появляются новые положительные носители тока, а при обработке во влажной атмосфере - как положительные $\left(\mathrm{H}^{+}\right)$, так и отрицательные $\left(\mathrm{OH}^{-}\right)$носители тока. По мере поглощения водорода электропроводность $\mathrm{Sr}_{2} \mathrm{CuO}_{3}$ уменьшается. Причиной является уменьшение подвижности основных носителей тока (дырок) вследствие увеличения дефектности решетки в связи с внедрением в нее ионов водорода. Резкое изменение электрофизических свойств при смене окислительной атмосферы на водород или влажную атмосферу, связанное с проникновением водорода или продуктов диссоциации воды в структуру $\mathrm{Sr}_{2} \mathrm{CuO}_{3}$, свидетельствует о высокой подвижности этих ионов при температурах $>70^{\circ} \mathrm{C}$.

Благодарности/Acknowledgements. Рентгенобазовый анализ образиов выполнен на оборудовании центра коллективного пользования "Испьтательный иентр нанотехнологий и перспективных материалов" ИФМ урО РАН. Работа выполнена в рамках государственного задания МИНОБРНАУКИ России по темам "Давление», № АААA-A18-118020190104-3; «Спин», № AAAA-A18-118020290104-2. / X-ray phase analysis of the samples was carried out on the equipment of the Collaborative Access Center "Testing Center of Nanotechnology and Advanced Materials" of the IMF UrB RAS. The work was performed within the framework of the state assignment of the Ministry of Education and Science of Russia on the themes "Pressure", No. AAAA-A18-118020190104-3; "Spin", No. AAAA-A18-118020290104-2.

\section{Литература/References}

1. Z. Hiroi, M. Takano, M. Azuma, Y. Takeda. Letters to nature. 364, 315 (1993). Crossref

2. Q. Q. Liu, H. Yang, Y. Yu, L.X. Yang, R.C. Yu, F. Y. Li, C. Q. Jin, S. Uchida. Physica C. 463-465, 100 (2007). Crossref

3. A. K. Ivanov-Shits, I. V. Murinю Ionica of solid state. V. 2. Saint Petersburg State University, Saint-Petersburg (2000) 890 p. (in Russian) [А. К. Иванов-Шиц, И. В. Мурин. Ионика твердого тела. Т. 2. Санкт-Петербургский государственный университет, Санкт-Петербург (2000) 890 c.]

4. W. Chen, K. He, Y. Wang, H. Chan, Z. Yan. Sci. Rep. 3, 3149 (2013). Crossref

5. Yu. M. Baikov. Phys. Solid State. 42 (6), 1026 (2000). $\underline{\text { Crossref }}$

6. I.B. Bobylev, S. V. Naumov, N.A. Zyuzeva. Phys. Solid State. 55 (8), 1602 (2013). Crossref

7. I. B. Bobylev, S.V. Naumov, N.A. Zyuzeva. Phys. Met. Metallogr. 118 (7), 671 (2017). $\underline{\text { Crossref }}$

8. M.D. McCluskey, M.C. Tarun, S.T. Teklemichael. J. Mater. Res. 27 (17), 2190 (2012). Crossref

9. H. Y. Noh, J. Kim, J.-S. Kim, M. J. Lee, H. J. Lee. Crystals. 9 (2), 75 (2019). Crossref

10. I. B. Bobylev, S. V. Naumov, N.A. Zyuzeva. Phys. Solid State. 58 (9), 1744 (2016). Crossref

11. I. B. Bobylev, S. V. Naumov, N. A. Zyuzeva, S. V. Telegin. Phys. Met. Metallogr. 119 (12), 1175 (2018). Crossref

12. T. Motoki, Y. Yanai, K. Nunokawa, S. Gondo, S. Nakamura, J. Shimoyama. Supercond. Sci. Technol. 33 (3), 034008 (2020). Crossref

13. V. P. Gorelov, V. B. Balakireva, A. V. Kuz'min, S. V. Plaksin. Inorganic materials. 50 (5), 495 (2014). $\underline{\text { Crossref }}$

14. S. N. Shkerin, M.V. Kuznetsov, N.A. Kalashnikova. Russian J. of Electrochem. 39 (6), 591 (2003). Crossref 
15. R. Smith. Semiconductors. Moscow, Mir (1982) 558 p. (in Russian) [Р. Смит. Полупроводники. Москва, Мир (1982) 558 c.]

16. M. A. Augustyniak-Jablokow, Yu. V. Yablokov, I. JacynaOnyszkiewicz, T. A. Ivanova, V.A. Shustov. Acta Phys. Pol. A. 112 (3), 523 (2007). Crossref

17. S. V. Telegin, A. Yu. Zuev, S. V. Naumov, E.I. Patrakov, D.S. Tsvetkov. Journal of Chemistry. 2017, 3057873 (2017). Crossref

18. S.S. Gorelik, M. Ya. Dashevskiy. Material science of semiconductors and dielectrics. Moscow, Metallurgiya (1988) 375 p. (in Russian) [С. С. Горелик, М.Я. Дашевский. Материаловедение полупроводников и диэлектриков. Москва, Металлургия (1988) 375 с.]
19. E. K. Shalkova, Yu.M. Baikov, T.A. Ushakova. Sverhprovodimost: Fizica, Khimiya, Tehnika. 5 (1), 24 (1992). (in Russian) [Е.К. Шалкова, Ю.М. Байков, Т. А. Ушакова. СФХТ. 5 (1), 24 (1992).]

20. N. A. Zyuzeva, I. B. Bobylev, S. V. Naumov, E. P. Romanov. Phys. Met. Metallogr. 115 (6), 547 (2014). Crossref

21. R. Zhao, M. J. Goringe, S. Myhra, P.S. Turner. Philosophical Mag. A. 66 (4), 491 (1992). Crossref

22. T. Hirata. Phys. Stat. Sol. (a). 156 (2), 227 (1996). Crossref

23. V.A. Zelenskiy, M.I. Alymov, A.B. Ankudinov, I. V. Tregubova. Perspektivnye materialy. 6 (6), 83 (2009). (in Russian) [В.А. Зеленский, М.И. Алымов, А.Б. Анкудинов, И.В. Трегубова. Перспективные материалы. 6 (6), 83 (2009).] 